\title{
Perceptual implicit memory relies on intentional, load-sensitive processing at encoding
}

\author{
BRIAN T. CRABB and VERONICA J. DARK \\ Iowa State University, Ames, Iowa
}

\begin{abstract}
In two experiments, we examined whether the encoding processes leading to perceptual implicit memory satisfied the intentionality and load insensitivity criteria for automaticity. Whether participants intended to process words or digits, in displays containing both, was manipulated in Experiment 1. Results showed an effect of intention on a subsequent perceptual identification task and a recognition task. Load (one, two, and four words) and exposure duration (1,000,600, and $200 \mathrm{msec}$ ) at encoding were manipulated in Experiment 2. Recognition was affected by both variables, but performance on the perceptual identification task was affected only by load. In both experiments, the results showed that controlled (intentional, load-sensitive) processing of words at encoding is essential for later perceptual implicit memory. That is, the encoding processes leading to perceptual implicit memory fail both criteria of automaticity.
\end{abstract}

The attention literature distinguishes between controlled and automatic processing (e.g., Hasher \& Zacks, 1979; Kahneman \& Treisman, 1984; Schneider, Dumais, \& Shiffrin, 1984; Shiffrin \& Schneider, 1977). According to this conceptualization, controlled processes are under the direct control of the individual, and they are capacity limited (i.e., they take up processing resources/working memory), whereas automatic processes are not under the direct control of the individual and they are capacity free (i.e., they do not take up processing resources/working memory). The ability to consciously recollect information presented during a prior episode requires controlled processing of the information at encoding (e.g., Fisk \& Schneider, 1984; Shiffrin \& Schneider, 1977). Indeed, in most dual-process models of information processing, controlled processing at encoding is necessary for explicit learning and memory (e.g., Cowan, 1995; Hasher \& Zacks, 1979; Shiffrin \& Schneider, 1977).

The implicit memory literature distinguishes between two types of test on the basis of whether the test is as-

Experiment 1 and portions of Experiment 2 were completed as part of the first author's master's thesis. Portions of both experiments were presented at the May 1998 meeting of the Midwestern Psychological Association, and the full Experiment 1 was presented at the November 1998 meeting of the Psychonomic Society. We thank Brenda Crawford, Sarah Daggett, Cederick Dark, and Jessie Everts for their assistance in collecting the data. We thank Peter Graf, Neil Mulligan, and Tim Curran for comments on an earlier version of this paper. Correspondence concerning this article may be addressed either to B. T. Crabb, Department of Psychology, MS 9089, Western Washington University, 516 High Street, Bellingham, WA 98225 or to V. J. Dark, Department of Psychology, Iowa State University, Ames, Iowa 50011-3180 (e-mail: brian.crabb@wwu.edu or vjdark@iastate.edu).

-This article was accepted by the previous editorial team. sumed to reflect conceptual or perceptual processing during encoding (e.g., Roediger, 1990). Conceptual implicit memory tests are affected by many of the same conceptual variables (e.g., the generation effect; Blaxton, 1989) that affect explicit memory tests. In contrast, perceptual implicit memory tests are unaffected by these variables but are affected by more sensory-based variables (e.g., font and modality; Graf \& Ryan, 1990; Jacoby, Levy, \& Steinbach, 1992). Whereas the necessity of controlled processing at encoding has been demonstrated for conceptual implicit memory tests (e.g., Mulligan, 1997; Mulligan \& Hartman, 1996), some researchers have posited that for perceptual implicit memory, controlled processing at encoding either is not as important (Jacoby, Lindsay, \& Toth, 1992; Jacoby, Toth, \& Yonelinas, 1993) or is not necessary at all (Mack \& Rock, 1998; Parkin \& Russo, 1990; Shiffrin, 1997). The present study was conducted to examine whether controlled processing at encoding is necessary for perceptual implicit memory.

Because the distinction between automatic and controlled processing depends jointly on the dimensions of intentional control and limited capacity, empirical investigations of whether or not some process is automatic often manipulate intention, a variable related to direct control, and load, a variable related to capacity. Yantis and Jonides (1990) captured this fact by referring to the intentionality criterion and the load insensitivity criterion of automaticity. The intentionality criterion states that automatic processes are not under the direct conscious control of the individual; automatic processes always occur, even when the individual is not intending them to occur. Thus, automatic processes satisfy the intentionality criterion, but controlled processes do not. The load insensitivity criterion states that automatic processes are not affected by increases in the amount of sen- 
sory information to be processed (the load). Thus, automatic processes satisfy the load insensitivity criterion, but controlled processes do not.

The goal of the present study was to test whether the kind of encoding that is tapped by a perceptual implicit memory test satisfies the intentionality and load insensitivity criteria of automaticity. Two experiments were conducted. Experiment 1 used a focused-attention task to test for the satisfaction of the intentionality criterion. Experiment 2 used a perceptual load manipulation in a divided-attention task to test for the satisfaction of the load insensitivity criterion. In both experiments, the stimuli of interest were words, and performance on a perceptual identification task provided the measure of perceptual implicit memory. Perceptual identification was used because it is considered to be the most process pure (i.e., least likely to be influenced by conceptual processing) of the perceptual implicit memory tests (Roediger, 1990). Also, in both experiments, explicit memory was measured by an old/new recognition test. This provided the opportunity to determine whether the manipulated variables had the appropriate effect on controlled processing at encoding and also allowed for an assessment of the extent to which the manipulations affected perceptual implicit memory tests and explicit memory tests in a similar or a different manner.

\section{EXPERIMENT 1}

Two different strategies have been employed in testing whether or not the encoding necessary for implicit memory satisfies the intentionality criterion. Both strategies use a focused-attention task in which participants attempt to process only a subset of the available information. That is, some information is defined as attended, or to-be-processed, and some is defined as unattended, or to-be-ignored. The first strategy follows an existence proof logic and simply assesses whether there is any evidence for implicit memory for unattended stimuli (e.g., Eich, 1984; Mack \& Rock, 1998). According to this strategy, if there is reliable implicit memory for unattended stimuli, implicit memory must reflect automatic encoding. The second strategy follows a direct comparison logic and assesses whether the levels of implicit memory for attended versus unattended stimuli differ (e.g., Crabb \& Dark, 1999; Hawley \& Johnston, 1991; MacDonald \& MacLeod, 1998). If implicit memory is the result of automatic processing at encoding, the instructions to attend to versus to ignore stimuli at encoding should not affect implicit memory test performance.

Eich (1984) used the first strategy and reported reliable implicit memory for unattended words in a dichotic listening task. Although participants demonstrated no explicit memory for the unattended words on a subsequent recognition test, they did demonstrate implicit memory for the unattended words on a spelling test (a conceptual implicit memory test). Eich concluded that the memory was the result of automatic processing of the unattended words. However, aspects of the design of Eich's study (e.g., lack of counterbalancing and repeated presentations of the stimuli) have been criticized (e.g., Cowan, 1995; Holender, 1986), and the findings have been replicated only when the presentation rate on the attended channel was sufficiently slow to allow for the possibility of switching attention between the attended and the unattended channels (Wood, Stadler, \& Cowan, 1997).

Mack and Rock (1998, chap. 8) also employed the first strategy and found evidence of perceptual implicit memory for unattended words. In what they termed an inattention condition, participants were required to make a judgment about which of two arms of a cross was longer. On the first two trials, the cross was displayed parafoveally for $200 \mathrm{msec}$. On the third trial, a word was presented foveally at the same time that the cross was presented. Even though the word was presented foveally and for a relatively long period of time, over $60 \%$ of the participants reported not seeing anything but the cross. Yet they were more likely to use the word to complete a word stem presented immediately after the third trial. Mack and Rock concluded that unattended words are unconsciously perceived and that unconscious perception can affect performance on a subsequent test. However, procedural features of the study (e.g., the immediate test, the small stimulus set, the use of just one implicit memory trial, and the fact that baseline observations were obtained under very different circumstances) make it difficult to generalize the findings to standard perceptual implicit memory tests.

Hawley and Johnston (1991, Experiment 2) followed the second strategy in testing the extent to which the encoding tapped in an implicit memory test satisfied the intentionality criterion. Participants saw 33- and 66-msec displays in which a word was centered between two digits (e.g., 6 wORD 2). Level of attention, or amount of intentional controlled processing, was manipulated by having different encoding tasks: One third of the participants always reported the word (100\% attention to the words), one third always reported the sum of the digits $(0 \%$ attention to the words), and one third did one or the other, depending on the cue that occurred after stimulus offset (50\% attention to the words). Results on a subsequent word identification task showed better performance for words originally presented at the longer duration and under higher levels of attention. Hawley and Johnston concluded that perceptual implicit memory varied as a function of intentional controlled processing of the stimuli.

Two other studies in which the second strategy was followed also showed that attending to, or intentionally processing, one stimulus rather than another at encoding results in reliable perceptual implicit memory for the attended stimulus, with no evidence of perceptual implicit memory for the unattended stimulus (Crabb \& Dark, 1999; MacDonald \& MacLeod, 1998, Experiment 3). In both studies, words were presented two at a time during encoding. MacDonald and MacLeod found that attended words (presented in a to-be-reported color at encoding) 
were read more quickly in a later speeded reading task than were either unattended words (presented in the nonreported color at encoding) or new words (not presented at encoding), which did not differ. Crabb and Dark instructed participants to report a spatially cued word and any other word that had been seen in the briefly presented word pair. They classified words as attended if they were reported. They found reliable perceptual implicit memory on both a word stem completion task and a perceptual identification task for attended words, but not for unattended words. In both studies, the authors concluded that controlled processing at encoding was necessary for later perceptual implicit memory.

Although there is no theoretical reason why the two different strategies should yield conflicting results, they did. We note that the two studies claiming to demonstrate implicit memory without controlled processing at encoding used tasks in which the attentional status of a stimulus (attended or unattended) was correlated across trials with its location (Mack \& Rock, 1998) or channel (Eich, 1984), whereas in two of the three studies making the opposite claim (Crabb \& Dark, 1999; MacDonald \& MacLeod, 1998), attentional status and stimulus location were uncorrelated. It is possible that the relationship between location and attentional status contributed to the different pattern of results. Although Hawley and Johnston (1991) presented the to-be-attended information at the same location on each trial, data limitations in processing may have occurred as a result of the short exposure durations (e.g., even with $50 \%$ attention at $33 \mathrm{msec}$, there was no reliable evidence of implicit memory). Therefore, in Experiment 1, we examined the intentionality criterion under conditions in which attentional status was correlated with location at encoding and exposure duration was relatively long $(600 \mathrm{msec})$. If the encoding processes leading to perceptual implicit memory fail the intentionality criterion under such conditions, the position that attention, or controlled processing, at encoding is essential for perceptual implicit memory would be strengthened.

In Experiment 1, we used a modified version of a procedure originally used by Fisk and Schneider (1984) to determine the role of controlled and automatic processing at encoding on a later explicit memory test. Their participants engaged in three tasks: an encoding task, a filler task, and a frequency estimation task (a measure of explicit long-term memory). In the initial encoding task, the participants viewed a series of briefly presented displays in which centrally presented three-letter words were surrounded by two digits and two pound signs. Controlled processing was manipulated by having the participants respond to different aspects of the displays. In the word condition, the instructions were to ignore the digits and to respond with a keypress whenever a vehicle name was presented; it was assumed that controlled processing would be directed only to the words. In the digit condition, the instructions were to ignore the words and to respond with a keypress whenever one of two tar- get digits was presented; it was assumed that controlled processing would be directed only to the digits. After completing the encoding task and the filler task, the participants estimated the frequency of nonvehicle words that had been presented between 0 and 20 times during the encoding task; the frequency estimates were used to compute word recognition performance. Results indicated high recognition for the participants in the word condition and chance recognition for those in the digit condition.

The procedure in Experiment 1 was similar to the word and digit conditions of Fisk and Schneider (1984), except that the dependent variable was either perceptual identification or old/new recognition accuracy. Also, each critical word was presented only once during the encoding task. The relationship between intention at encoding and perceptual implicit memory was examined, following the logic of both strategies described earlier. First, we determined whether there was a reliable effect of encoding condition on implicit memory - the absence of an effect supporting the role of automatic processing at encoding and the existence of an effect supporting the role of controlled processing at encoding. Second, we determined whether implicit memory was reliably greater than chance for words in the digit condition. Because it is well established that explicit memory requires controlled processing at encoding (e.g., Fisk \& Schneider, 1984), encoding condition should affect recognition test performance, and there should not be above-chance recognition performance in the digit condition.

\section{Method}

\section{Participants and Design}

The participants were 192 undergraduate students enrolled in introductory psychology courses. They received extra credit for their participation. All the participants reported normal or corrected-tonormal vision and fluency in English. Forty-eight participants were assigned to each of four between-subjects conditions derived from the orthogonal combination of the two encoding conditions (digit or word) and the two memory tests (recognition or perceptual identification).

\section{Stimuli}

A pool of 120 words, 3-11 letters in length, selected from Roediger, Weldon, Stadler, and Riegler (1992, Experiment 3), was divided into six sets of 20 words each in such a way that the sets were approximately equal along the dimension of reportability on a perceptual identification test, as assessed by an earlier study (Crabb \& Dark, 1999). For each participant, 60 of these words appeared in the initial encoding task and as old words in the subsequent memory test. The other 60 words were used as the baseline (or new) words in the memory test. The old/new status of each word was counterbalanced over participants.

Twelve vehicle names, 3-10 letters in length, were presented as part of the encoding task and functioned as the targets for the word condition. They were not presented in the memory test. Another 200 words, also 3-11 letters in length, served as filler words in the encoding task and were not presented in the memory test. Whereas the vehicle names were repeated throughout the encoding task, all the other words appeared only once. Two randomly selected digits (drawn from the set 
consisting of 2, 3, 5, 6, 7, 8, and 9) presented at the start of each sequence functioned as the targets for the digit condition.

\section{Procedure}

The participants were seated approximately $40 \mathrm{~cm}$ from the monitor of a Zenith 386 computer running Micro Experimental Laboratory software (Schneider, 1988). All the stimuli were presented as white characters on a black background, using the standard font. The participants engaged in three tasks: (1) the initial encoding task, (2) a 5-min math test, and (3) a memory test, either recognition or perceptual identification. The participants were told that the three tasks were being used to test the cognitive abilities of college students of various ages.

Encoding task. The participants were presented 20 display sequences, with each sequence consisting of 19 words and 76 digits. The 1st sequence served as practice to ensure that the participants understood and were able to perform the task. The next 4 sequences and the last 5 sequences were buffer sequences. The middle 10 sequences were the critical sequences, in which the to-be-tested words were presented. Each sequence consisted of 2 digits (the target set for the digit detection task) for $3 \mathrm{sec,} \mathrm{a} \mathrm{fixation} \mathrm{dot} \mathrm{for}$ $500 \mathrm{msec}$, a display of Xs for $300 \mathrm{msec}$, a display of Ys for $300 \mathrm{msec}$, and then a series of displays each consisting of a centered 3-11 letter word and four elements positioned to form a rectangle around the word (see Figure 1). The four elements consisted of 2 digits on one diagonal of the rectangle and two pound signs on the other diagonal and were presented four positions to the left and right of center. The particular diagonal containing the digits alternated between displays. Each display remained on for $300 \mathrm{msec}$. Each word was presented for the duration of two displays (or $600 \mathrm{msec}$ total). Following the final word, a display of Xs was presented for $300 \mathrm{msec}$, to serve as a mask.

Examples of the various types of displays are shown in Figure 1. In each of the sequences, the first and the last 2 words were buffers. Of the other 15 words, 3 were vehicle names (i.e., targets for the word condition). The words presented immediately after vehicle words were buffers, to allow cognitive recovery from responses in the word condition. Digit targets (i.e., digits from the display beginning the sequence) occurred with the words presented immediately before the vehicle words. On the 10 critical sequences, the remaining 6 words were the critical words that were later tested. The target sets (consisting of a vehicle name and the immediately preceding and subsequent words) were randomly distributed within the sequence, with the provision that they did not appear in the first or the last two displays. ${ }^{1}$

The participants in the word condition were instructed to ignore the digits and to press the space bar whenever they detected a word that was a vehicle name. The participants in the digit condition were instructed to ignore the words and to press the space bar whenever they detected the presence of one of two digits presented at the start of the sequence. Both accuracy and latency were emphasized. No feedback was given.

Math task. Following the encoding task, the participants engaged in a math task lasting approximately $5 \mathrm{~min}$. The participants determined whether simple arithmetic equations (e.g., $2+2=5$ ) were true. Each problem was presented on the computer screen, and the participants responded by keypress as quickly and accurately as possible ("1" for true and " 2 " for false). The next problem was presented immediately after the response. The math task served as a distraction task designed to clear working memory and to disconnect the subsequent memory test from the encoding task. Following the math task, the participants engaged in either the recognition or the perceptual identification test.

Recognition. In the recognition test, each trial began with a fixation point $(++)$ for $500 \mathrm{msec}$. Following fixation, a word was presented. The participants were instructed to indicate by keypress whether or not the word had appeared in the encoding task (the let- ter "A" key for old and the letter "L" key for new). The word remained on the screen until a response was made. There were 120 trials, 60 with old words from the encoding task and 60 with new, or unstudied, words drawn from the set of words described in the Stimuli section.

Perceptual identification. In the perceptual identification test, each trial began with a fixation point $(++)$ for $500 \mathrm{msec}$. Following fixation, a word was presented for $33 \mathrm{msec}$. Each word was followed by a row of Xs, which remained on until a response was made. The participants were instructed to name aloud the word that had preceded the Xs. A condenser microphone placed approximately $10 \mathrm{~cm}$ from the participant's mouth was used to register the occurrence of the response; accuracy was recorded by an experimenter. There were 130 trials. Because perceptual identification is a difficult task, the first 10 trials were inserted as practice to give the participants some experience before they encountered the critical trials. Of the words presented on the remaining 120 trials, 60 were the old words from the encoding task, and 60 were new words.

The entire procedure took approximately $30 \mathrm{~min}$. The participants were debriefed at the end of the experiment.

\section{Results}

An alpha level of .05 was used for all analyses.

\section{Encoding Task}

Performance at encoding was measured as the proportion of hits. A hit was defined as a detection response made to a target within $1,200 \mathrm{msec}$ of the onset of the target. The proportion of hits in the digit condition was $.598(S E=.023)$. The proportion of hits in the word condition was $.971(S E=.030)$. As was expected on the basis of stimulus duration (300 msec for digits and $600 \mathrm{msec}$ for words) and number of relevant items per display (two for digits and one for words), the digit detection task was more difficult than the vehicle detection task $[t(190)=16.43, S E=.023, p<.001]$. If it is assumed that the participants did as instructed, the relatively low performance on the digit task suggests that the task consumed most of the participants' controlled processing resources, so that the words presented during the digit detection task received little controlled processing.

\section{Recognition}

The proportion of old responses to old words (hits) and the proportion of old responses to new words (false alarms) are shown in the top half of Table 1 for each of the encoding conditions. Recognition memory was indexed as a difference score by subtracting the proportion of false alarms from the proportion of hits. The difference scores also are shown in the top half of Table 1. Recognition memory for words was higher when the intention at encoding was to process the words rather than the digits $[t(94)=8.32, S E=.022, p<.001]$, replicating a well-established finding that controlled processing affects explicit memory. However, a one-tailed $t$ test against zero showed reliable recognition memory in the digit condition $[t(47)=1.77, S E=.013, p=.041]$, as well as in the word condition $[t(47)=11.49, S E=.018, p<.001]$. Reasons for this somewhat unexpected finding will be considered later. 


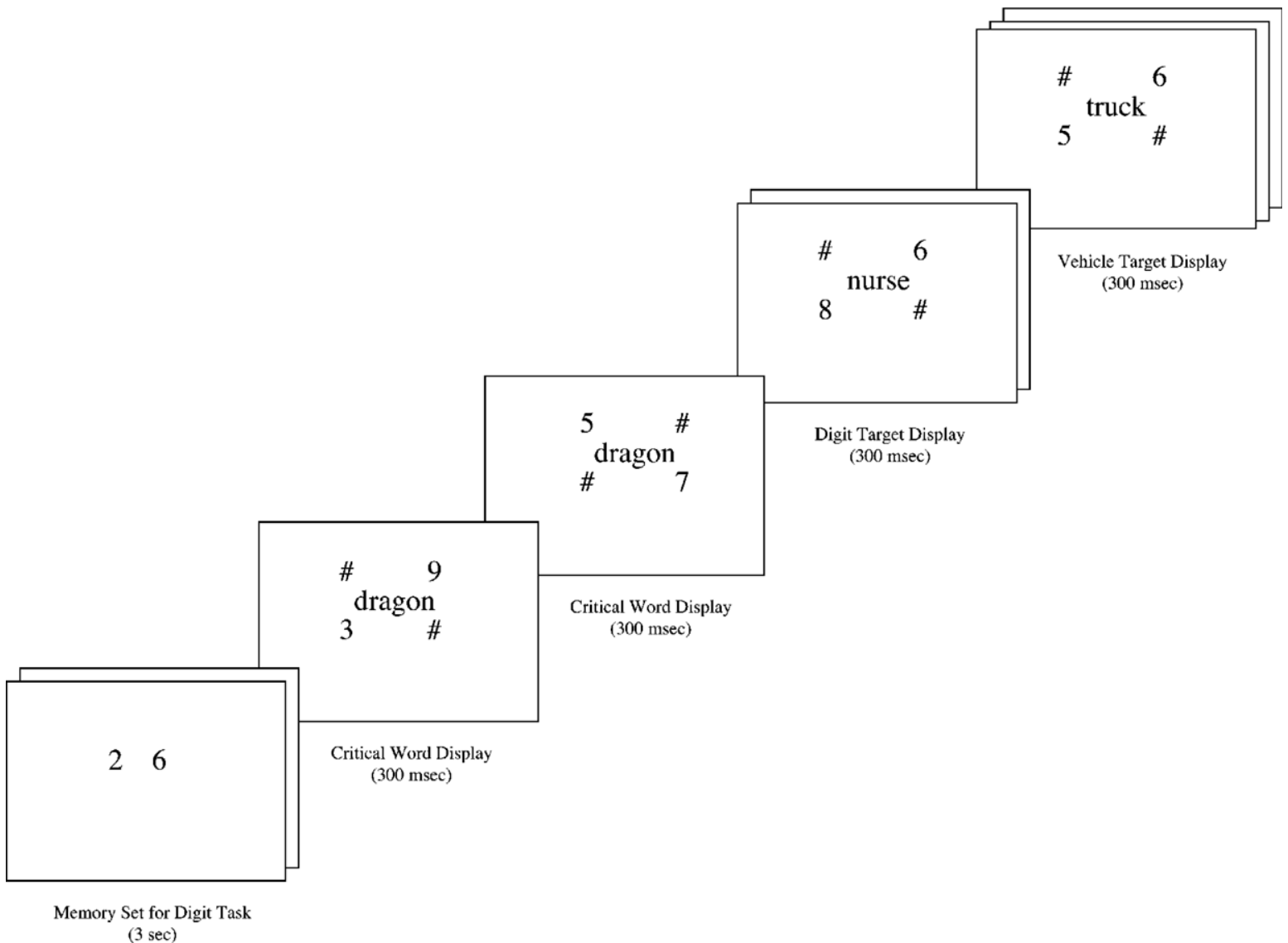

Figure 1. Examples of displays in the encoding task in Experiment 1.

\section{Perceptual Identification}

The proportion of old words that were identified and the proportion of new words that were identified are shown in bottom half of Table 1 for each of the encoding conditions. Perceptual implicit memory was indexed as a difference score by subtracting the proportion of new words identified from the proportion of old words iden- tified. The difference scores are also shown in the bottom half of Table 1. Perceptual implicit memory was higher when the intention at encoding was to process the words rather than the digits $[t(94)=3.13, S E=.016, p<$ $.001]$. As with the recognition results, one-tailed $t$ tests against zero showed reliable perceptual implicit memory in the digit condition $[t(47)=1.86, S E=.012, p=.035]$,

Table 1

Performance on the Memory Tasks in Experiment 1 as a Function of Intention at Encoding and Type of Word

\begin{tabular}{|c|c|c|c|c|c|}
\hline \multirow[b]{3}{*}{ Memory Task } & \multirow[b]{3}{*}{ Type of Word } & \multicolumn{4}{|c|}{ Intention } \\
\hline & & \multicolumn{2}{|c|}{ Digits } & \multicolumn{2}{|c|}{ Words } \\
\hline & & $M$ & $S E$ & $M$ & $S E$ \\
\hline \multirow[t]{3}{*}{ Recognition } & Old $^{a}$ & .44 & .02 & .53 & .02 \\
\hline & $\mathrm{New}^{\mathrm{b}}$ & .42 & .02 & .32 & .02 \\
\hline & Difference & $.02 *$ & .01 & $.21 *$ & .02 \\
\hline \multirow[t]{3}{*}{ Perceptual identification } & Old & .50 & .03 & .59 & .03 \\
\hline & New & .48 & .03 & .52 & .03 \\
\hline & Difference & $.02 *$ & .01 & $.07 *$ & .01 \\
\hline
\end{tabular}

${ }^{a}$ For recognition task performance, this is the proportion of old responses to old words (hits). bFor recognition task performance, this is the proportion of old responses to new words (false alarms). *Reliably different from zero, $p<.05$. 
as well as in the word condition $[t(47)=7.12, S E=.010$, $p<.001]$.

\section{Discussion}

Experiment 1 used a modification of the focusedattention procedure of Fisk and Schneider (1984) to test whether the encoding necessary for perceptual implicit memory satisfied the intentionality criterion. If perceptual implicit memory, as indexed by the perceptual identification difference score, was due solely to automatic encoding processes, the intention of the participants at encoding should not have affected the level of perceptual implicit memory, and there should have been perceptual implicit memory for unattended words. If perceptual implicit memory was due solely to controlled encoding processes, intention should have affected the level of perceptual implicit memory, and there should not have been reliable perceptual implicit memory for unattended words. The results fell in between the two extreme possibilities, in that there was an effect of intention on perceptual implicit memory but there also was evidence of some implicit memory for unattended words. The very same pattern was found for recognition memory, a type of explicit memory.

Although implicit memory for words was higher when words were intentionally processed, there was reliable implicit memory for unattended words (i.e., words presented when the digits were intentionally processed). If only the implicit memory data are considered, therefore, it could be argued that while the encoding necessary for implicit memory does not appear to be automatic in the strong sense, it does appear to be partially automatic. Unlike strongly automatic processes, which do not require controlled processing and are unaffected by it, partially automatic processes do not require controlled processing but can be enhanced by the addition of controlled processing (Kahneman \& Treisman, 1984). When the implicit memory data are considered in conjunction with the explicit memory data, however, an alternative interpretation is suggested: At least some words in the digit condition received some amount of controlled processing, and the very low, but reliable, level of implicit and explicit memory for unattended words reflects this controlled processing. Because this interpretation handles the results of both the implicit and the explicit memory tests, it is more parsimonious.

Fisk and Schneider (1984, Experiment 2) also found evidence of low, but reliable, explicit (recognition) memory for words presented under conditions in which only the digits should have been attended. They suggested that even though the difficulty of the digit detection task required participants to devote all their controlled processing resources to that task, the participants occasionally may have switched attention from the digits to the words; that is, they acknowledged the possibility that the procedure did not completely prevent controlled processing of at least some words. The possibility that words in the digit condition received some controlled processing is even more likely in the present experiment. In Fisk and Schneider, all the words were 3 letters in length, and therefore, no letters ever occurred adjacent to the digits, which occurred two positions to the left and right of center. Our words varied in length from 3 to 11 letters, so that letters occasionally occurred directly above/below or even to the left of the leftmost digits or to the right of the rightmost digits. In our view, the more parsimonious explanation of the explicit memory and implicit memory results as a whole is that controlled processing was occasionally directed to words in the digit condition and that controlled processing at encoding is necessary for implicit memory, as well as for explicit memory. ${ }^{2}$

Experiment 1 provides further support for past studies demonstrating that words must receive some minimal amount of controlled processing at encoding to support later perceptual implicit memory (e.g., Crabb \& Dark, 1999; Hawley \& Johnston, 1991; MacDonald \& MacLeod, 1998). In addition, the results show that the findings of these previous studies do not depend on there being spatial uncertainty in the location of the to-be-attended stimuli or on the use of data-limited displays. In the present research, we used a relatively long display time $(600 \mathrm{msec})$ for words displayed at a single location and still demonstrated that unless words are attended (intentionally processed) at encoding, little perceptual implicit memory will be demonstrated. That is, the encoding processes leading to perceptual implicit memory fail the intentionality criterion of automaticity. In Experiment 2, we addressed the load insensitivity criterion.

\section{EXPERIMENT 2}

Load refers to the amount of information in a situation. Controlled processing is load sensitive, and automatic processing is load insensitive. Two forms of load can be manipulated at encoding: cognitive load and perceptual load. Cognitive load at encoding refers to the amount of information that must be maintained in working memory while some stimulus is simultaneously encoded. Perceptual load refers to the amount of sensory information that is presented simultaneously during encoding (e.g., the number of items in the visual display).

Researchers interested in whether the encoding processes underlying implicit memory are automatic have investigated the load insensitivity criterion with manipulations of cognitive load (e.g., Jacoby et al., 1992; Mulligan, 1997; Mulligan \& Hartman, 1996; Parkin, Reid, \& Russo, 1990; Parkin \& Russo, 1990; Smith \& OscarBerman, 1990). Typically, in such research, participants either did or did not maintain a distractor list in working memory while they encoded a list of critical words. The underlying assumption was that a distractor list would consume controlled processing resources, leaving fewer resources to be used for encoding the critical list. The findings were that a distractor list produced lower mem- 
ory for the critical words when memory was assessed with explicit memory tests and with conceptual implicit memory tests (showing the importance of controlled processing for those types of test) but that there was little or no effect of a distractor list when memory was assessed with perceptual implicit memory tests (e.g., Mulligan, 1997; Parkin et al., 1990). That is, the encoding necessary for perceptual implicit memory appeared to satisfy the load insensitivity criterion when conceptual load was manipulated.

The question of concern in Experiment 2 was whether the encoding necessary for perceptual implicit memory would satisfy the load insensitivity criterion when perceptual load was manipulated. At encoding, the participants engaged in a vehicle detection task in which words were presented one, two, or four at a time. Because controlled processing is limited in capacity, the more words that there are to be processed, the fewer controlled processing resources there are that can be devoted to each word. Because the conceptual processing underlying performance on explicit memory tests requires controlled processing, we expected load to affect recognition memory. The question was whether the processing underlying performance on perceptual implicit memory also requires controlled processing. If it is an automatic process, the amount of information to be encoded should have no effect on the level of implicit memory found on a later test; that is, the processing would be load insensitive. If the amount of information that must be encoded does affect the level of implicit memory found on a later test, the encoding necessary for later perceptual implicit memory does not meet the load insensitivity criterion of automaticity.

We also varied exposure duration, with displays being presented for 200,600 , or $1,000 \mathrm{msec}$. We assumed that at longer durations, there would be opportunity for more controlled processing of each word, for controlled processing of more words, or for both. Because explicit memory depends on controlled processing, we expected that exposure duration would affect recognition memory with at least the higher loads. The question was whether or not it would affect perceptual implicit memory. Previous research suggested that no effect should be found (Jacoby \& Dallas, 1981; Neill, Beck, Bottalico, \& Molloy, 1990; von Hippel \& Hawkins, 1994). For example, when using an encoding task comparable to the one in Experiment 2, von Hippel and Hawkins found an effect of duration on recognition memory but no effect on perceptual implicit memory.

\section{Method}

\section{Participants and Design}

The participants were 180 undergraduate students enrolled in introductory psychology courses. They received extra credit for their participation. All the participants reported normal or corrected-tonormal vision and fluency in English. Thirty participants were assigned to each of six between-subjects conditions derived from the orthogonal combination of the three encoding durations $(1,000$,
600 , or $200 \mathrm{msec}$ ) and the two memory tests (recognition or perceptual identification). Encoding load (one, two, or four words) was manipulated within subjects.

\section{Procedure}

Except for the encoding task, the procedure was the same as that in Experiment 1. In addition, the equipment, software, and word stimuli were the same, except that an additional 208 words were added as fillers in the encoding task.

In the encoding task, the participants were presented 150 trials50 with one word, 50 with two words, and 50 with four words. Each trial consisted of a fixation point $(++)$ presented for $500 \mathrm{msec}$ and a display of words presented for $1,000,600$, or $200 \mathrm{msec}$, with an intertrial interval of 500,900, or 1,300 msec, respectively (yielding a constant stimulus onset asynchrony of $1,500 \mathrm{msec}$ ). Load 1 words were displayed in one of four locations: above, below, to the right of, or to the left of fixation. Load 2 words were displayed either above and below or to the left and to the right of fixation. The first and last 9 trials ( 3 of each load) were buffers. Of the middle 132 trials, 36 contained a vehicle name (12 at each load). Each vehicle name display was followed by a buffer trial, to allow cognitive recovery from the response. Each of the remaining 60 displays that did not contain a vehicle name (20 at each load) contained a critical word that was later tested on the recognition or the perceptual identification test. The critical words and the vehicle words were counterbalanced across participants in such a way that each appeared in each position and at each load an equal number of times. The participants were instructed to press the space bar as quickly as they could each time they detected a vehicle name. No feedback was given during the task.

\section{Results}

An alpha level of .05 was used for all the analyses.

\section{Encoding Task}

Performance at encoding was measured as the proportion of hits. A hit was defined as a detection response made to a vehicle name within $1,500 \mathrm{msec}$ of stimulus onset. The proportion of hits is shown as a function of encoding load (one, two, or four words) and exposure duration $(1,000,600$, or $200 \mathrm{msec})$ in Table 2 . An analysis of variance (ANOVA) with load as a within-subjects variable and exposure duration as a between-subjects variable showed a reliable main effect of encoding load $\left[F(2,354)=246.47, M S_{\mathrm{e}}=.009, p<.001\right]$, with a higher proportion of hits for lower loads, and a reliable main effect of exposure duration $\left[F(2,177)=13.17, M S_{\mathrm{e}}=.035\right.$, $p<.001]$, with a higher proportion of hits for longer durations. There was a reliable encoding load $\times$ exposure duration interaction $\left[F(4,354)=25.58, M S_{\mathrm{e}}=.009, p<\right.$

Table 2

Proportion of Vehicles Detected During the Encoding Task of Experiment 2 as a Function of Perceptual Load and Exposure Duration

\begin{tabular}{|c|c|c|c|c|c|c|}
\hline \multirow[b]{3}{*}{ Perceptual Load } & \multicolumn{6}{|c|}{ Exposure Duration (msec) } \\
\hline & \multicolumn{2}{|c|}{1,000} & \multicolumn{2}{|c|}{600} & \multicolumn{2}{|c|}{200} \\
\hline & $M$ & $S E$ & $M$ & $S E$ & $M$ & $S E$ \\
\hline Load 1 & .97 & .02 & .93 & .01 & .97 & .01 \\
\hline Load 2 & .94 & .02 & .90 & .01 & .86 & .02 \\
\hline Load 4 & .84 & .02 & .77 & .02 & .61 & .02 \\
\hline
\end{tabular}


.001]. The simple main effect of load was reliable at all exposure durations. Comparisons among the means, using Tukey's HSD test, showed that at the 200-msec duration, the proportion of hits differed reliably among all three levels of load but, for the two longer exposure durations, there was no reliable difference in the proportion of hits between Load 1 and Load 2 (HSD = .046). In other words, it appears that load had more of an impact at the shortest exposure duration, although the high level of performance on the task might suggest that the interaction was due to ceiling effects.

\section{Recognition}

As in Experiment 1, recognition memory was indexed as a difference score by subtracting the proportion of false alarms $[.27(S E=.02), .31(S E=.03), .31(S E=.03)$ for the 1,000-, 600-, and 200-msec conditions, respectively] from the proportion of hits. The difference scores are shown in Figure 2 as a function of encoding load and exposure duration. One-tailed $t$ tests against zero showed reliable recognition memory in all conditions (all $t \mathrm{~s} \geq$ 2.50). An ANOVA with load as a within-subjects variable and exposure duration as a between-subjects variable showed a reliable main effect of exposure duration $\left[F(2,87)=3.63, M S_{\mathrm{e}}=.052, p=.031\right]$. Comparisons among the means showed that recognition memory was greater for words displayed for $1,000 \mathrm{msec}$ than for words displayed for $200 \mathrm{msec}$ but that neither differed reliably from the recognition memory for words displayed for $600 \mathrm{msec}(\mathrm{HSD}=.080)$. There also was a reliable effect of encoding load $\left[F(2,174)=102.75, M S_{\mathrm{e}}=.012, p<\right.$
.001]. Comparisons among the means showed that recognition memory differed reliably among the three levels of load $(H S D=.039)$. The interaction was not reliable.

\section{Perceptual Identification}

As in Experiment 1, perceptual implicit memory was indexed as a difference score by subtracting the proportion of new words identified [.48 $(S E=.04), .52(S E=.03)$, and $.51(S E=.03)$ for the 1,000-, 600-, and 200-msec conditions, respectively] from the proportion of old words identified. The difference scores are shown in Figure 3 as a function of encoding load and exposure duration. One-tailed $t$ tests against zero showed reliable implicit memory for Load 1 at all durations, for Load 2 at 1,000 and $600 \mathrm{msec}$, and for Load 4 at $200 \mathrm{msec}$ (all $t \mathrm{~s} \geq$ 2.22). An ANOVA with load as a within-subjects variable and exposure duration as a between-subjects variable showed a reliable main effect of encoding load $[F(2,174)=$ $\left.6.55, M S_{\mathrm{e}}=.010, p=.002\right]$. No other effects were reliable (all $F_{\mathrm{S}}<1$ ). Comparisons among the means showed that the pattern was the same as that found for recognition: Perceptual implicit memory was greater for Load 1 words than for Load 4 words, but neither differed reliably from the level of perceptual implicit memory found for Load 2 words $(\mathrm{HSD}=.035)$.

\section{Discussion}

We manipulated perceptual load and exposure duration, variables that have been linked to controlled processing. As was expected, because they depend on controlled processing, target detection and recognition memory each

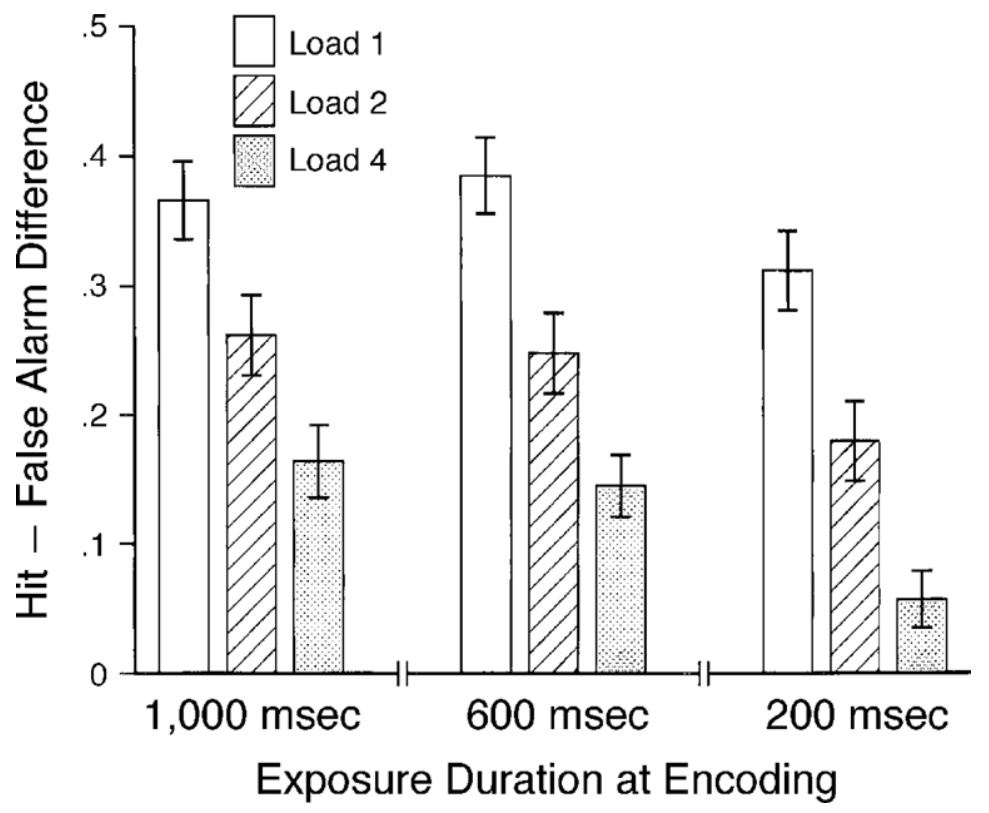

Figure 2. Difference scores (proportion of hits minus proportion of false alarms) on the recognition task in Experiment 2 as a function of load and exposure duration at encoding. Standard errors are also shown. 


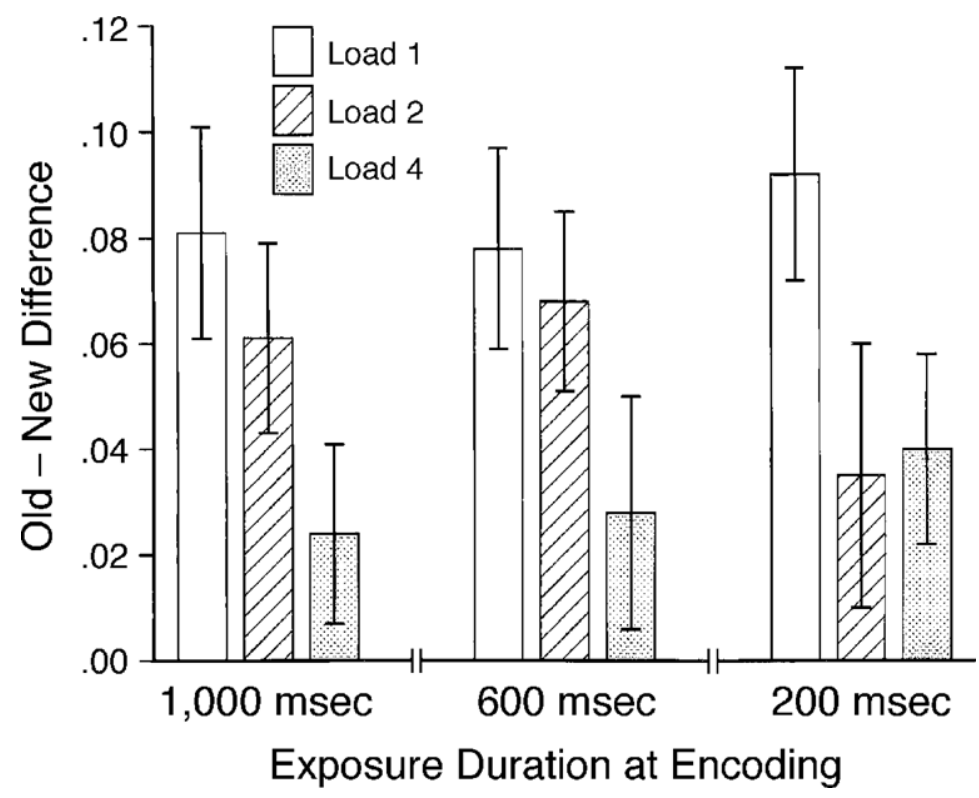

Figure 3. Difference scores (proportion of old words correctly identified minus the proportion of new words correctly identified) on the perceptual identification task in Experiment 2 as a function of load and exposure duration at encoding. Standard errors are also shown.

were affected by both variables. For both target detection and recognition, performance was lower when exposure duration was shorter, and performance was lower when the display contained more sensory information. In contrast, perceptual implicit memory was affected by perceptual load, but not by exposure duration. The results suggest that the encoding necessary for perceptual implicit memory requires controlled processing, because perceptual implicit memory was load sensitive, just as were recognition and target detection. However, the results also suggest that some forms of controlled processing affect only explicit, and not perceptual implicit, memory, because perceptual implicit memory was duration insensitive, whereas recognition and target detection were not.

The apparent conflict in the extent to which perceptual implicit memory depends on controlled processing has been noted in the literature. In order to make sense of the conflict, researchers investigating the role of controlled processing in implicit memory have suggested that controlled processing should be divided into at least two levels or kinds that capture the distinction between perceptual and conceptual processing: Lexical access is an early perceptually based stage of controlled processing, and temporal-spatial processing is a later more conceptually based controlled processing (e.g., Cowan, 1995; Mulligan, 1997; Weldon, 1991). According to this view, lexical access underlies performance on perceptual implicit memory tests, and temporal-spatial processing underlies performance on explicit memory tests and conceptual implicit memory tests.

Given that there are at least two types of controlled processing, the findings that exposure duration affects recognition, but not perceptual implicit memory (e.g., the present findings; Jacoby \& Dallas, 1981; Neill et al., 1990; von Hippel \& Hawkins, 1994), show that duration manipulations, at least those above some minimal threshold, ${ }^{3}$ affect the amount of temporal-spatial processing that a word receives at encoding, and not the early lexical access needed for perceptual implicit memory. On the basis of the findings that cognitive load at encoding affected explicit memory and conceptual implicit memory, but not perceptual implicit memory, Mulligan (1997; Mulligan \& Hartman, 1996) concluded that cognitive load affects only temporal-spatial processing. The present results allow an extension of the conclusion to include perceptual load, which does affect perceptual implicit memory. The conclusion would be that cognitive load and perceptual load influence different types of controlled processing and that perceptual load affects early lexical access. Thus, the encoding processes leading to perceptual implicit memory fail the load insensitivity criterion of automaticity, but only when perceptual load is manipulated.

\section{GENERAL DISCUSSION}

The present research was designed to determine whether the encoding processes that lead to perceptual implicit memory are automatic or controlled, as assessed by the load insensitivity and intentionality criteria of Yantis and Jonides (1990). Experiment 1 tested the intentionality criterion by manipulating whether or not participants attended to the stimuli at encoding. Experiment 2 tested the load insensitivity criterion by manipulating the perceptual load at encoding. 
The results of Experiment 1 provided support for a controlled processing position, because there was a reliable effect of intention at encoding on perceptual implicit memory. The results also provided some support for the partially automatic processing position, because there was reliable implicit memory for words in the digit condition, words that should not have received controlled processing. However, reliable recognition memory was found also for words in the digit condition. If it is assumed that explicit recognition memory results only from controlled processing at encoding (e.g., Fisk \& Schneider, 1984; Shiffrin \& Schneider, 1977), the latter finding shows that controlled processing of the words occasionally occurred during the digit condition. The results of Experiment 1 fit well with the findings of other studies (e.g., Bentin, Moscovitch, \& Nirhod, 1998; Crabb \& Dark, 1999; Hawley \& Johnston, 1991; MacDonald \& MacLeod, 1998) suggesting that an initial investment of controlled processing at encoding is essential for perceptual implicit memory. Thus, the conclusion from Experiment 1 is that the encoding processes leading to perceptual implicit memory fail the intentionality criterion of automaticity.

The perceptual load results of Experiment 2 also provided support for a controlled processing position. There was a reliable effect of perceptual load at encoding on both recognition and perceptual implicit memory. The effect on recognition, a measure of explicit memory, was expected, because explicit memory results from loadsensitive controlled processing at encoding (e.g., Shiffrin $\&$ Schneider, 1977). The similarity of the effect on perceptual implicit memory suggests that the same is true for perceptual implicit memory. Thus, the conclusion from Experiment 2 is that the encoding processes leading to perceptual implicit memory fail the load insensitivity criterion of automaticity.

The results of Experiment 2 concerning exposure duration add to the literature suggesting that there are different types of controlled processing. Although both are assumed to influence controlled processing, manipulation of exposure duration had a different effect on performance than did manipulation of perceptual load. Exposure duration affected both target detection and recognition, but it did not affect perceptual implicit memory. On the basis of this pattern, it appears that exposure duration affects a type of controlled processing that is not necessary for perceptual implicit memory. If a distinction is made between early lexical access and later temporal-spatial processing (e.g., Cowan, 1995; Mulligan, 1997; Weldon, 1991), exposure duration may influence the latter, but not the former. ${ }^{4}$

The implicit memory literature assumes that perceptual implicit memory tests reflect perceptual processing at encoding and that perceptual processing can be distinguished from conceptual processing (e.g., Roediger, 1990). The perceptual versus conceptual distinction is not the same as the distinction between automatic and controlled processing. The present results suggest that both perceptual and conceptual processing require controlled processing, or attention. Weldon (1991) and Mulligan (1997) distinguished between two types of controlled processing: lexical access and temporal-spatial processing. They suggested that perceptual implicit memory depends only on lexical access. However, recent studies suggest that while lexical access is necessary for later perceptual implicit memory, it might not be sufficient; other controlled processing must occur (Mulligan \& Hornstein, 2000; Stone, Ladd, Vaidya, \& Gabrieli, 1998).

Masson and MacLeod (1992) presented a related, but somewhat broader, view in which controlled processing at encoding can be roughly split into two phases: interpretive processing and elaborative processing. Interpretive processing is context-sensitive processing that enables one to gain an initial interpretation of a stimulus. Such processing is not restricted to orthographic or lexical processing and often will include semantic information, if that is necessary for a proper interpretation of the stimulus. Elaborative processing takes the established interpretation and relates it to previously stored memory representations. The framework posits that interpretive processing underlies implicit memory tasks and elaborative processing underlies explicit memory tasks.

The framework of Masson and MacLeod (1992) states that any processing that aids in the interpretation of a word at encoding will affect the level of later perceptual implicit memory for that word. Thus, semantic processing of a word at encoding could increase later perceptual implicit memory, but only if the semantic processing at encoding was a part of the initial interpretation of the word. In Experiment 2 and in the word condition in Experiment 1 , words were identified at encoding in a context in which a vehicle identification task was done. To fully interpret the words at encoding, the participants had to access the lexical unit for the word and to decide whether the word was a vehicle or not. Thus, manipulation of any variable that affected the ability of the participants to carry out either lexical access or the vehicle decision (or both) at encoding should have affected the level of later perceptual implicit memory. Intention to process (Experiment 1) and perceptual load (Experiment 2) were such variables. However, manipulation of any variable that affected the ability of the participants to carry out any further elaborative (or temporal-spatial) processing should not have affected the level of later perceptual implicit memory but should have affected the level of later explicit memory. Exposure duration (Experiment 2) was such a variable.

The present research adds to both the implicit memory and the word identification literatures. The research demonstrates that the interpretive encoding processes that underlie perceptual implicit memory are of a controlled processing nature. These processes are both intentional and perceptual-load sensitive. The present research also adds to knowledge about word identification. Other studies have demonstrated that word identification 
requires controlled processing (e.g., Herdman, 1992; McCann, Folk, \& Johnston, 1992). The present research shows that changes within the word identification system also require controlled processing. Indeed, all longterm changes in memory (whether measured with an explicit or an implicit memory test) appear to be the result of controlled processing at encoding (cf. Shiffrin \& Schneider, 1977).

\section{REFERENCES}

Bentin, S., Moscovitch, M., \& Nirhod, O. (1998). Levels of processing and selective attention effects on encoding in memory. Acta Psychologica, 98, 311-341.

Blaxton, T. A. (1989). Investigating dissociations among memory measures: Support for a transfer appropriate processing framework. Journal of Experimental Psychology: Learning, Memory, \& Cognition, 15, 657-668.

Cowan, N. (1995). Attention and memory: An integrated framework. New York: Oxford University Press.

Crabi, B. T., \& Dark, V. J. (1999). Perceptual implicit memory requires attentional encoding. Memory \& Cognition, 27, 267-275.

EIcH, E. (1984). Memory for unattended events: Remembering with and without awareness. Memory \& Cognition, 12, 105-111.

Fisk, A. D., \& Schneider, W. (1984). Memory as a function of attention, level of processing, and automatization. Journal of Experimental Psychology: Learning, Memory, \& Cognition, 10, 181-197.

GRAF, P., \& RYAN, L. (1990). Transfer-appropriate processing for implicit and explicit memory. Journal of Experimental Psychology: Learning, Memory, \& Cognition, 16, 978-992.

HASHER, L., \& ZACKS, R. T. (1979). Automatic and effortful processes in memory. Journal of Experimental Psychology: General, 108, 356-388.

HAWLEY, K. J., \& JoHnSTON, W. A. (1991). Long-term perceptual memory for briefly exposed words as a function of awareness and attention. Journal of Experimental Psychology: Human Perception \& Performance, 17, 807-815.

Herdman, C. M. (1992). Attentional demands of visual recognition in naming and lexical decision. Journal of Experimental Psychology: Human Perception \& Performance, 18, 460-470.

Hirshman, E., \& Mulligan, N. (1991). Perceptual interference improves explicit memory but does not enhance data-driven processing. Journal of Experimental Psychology: Learning, Memory, \& Cognition, 17, 507-513.

Hirshman, E., Trembath, D., \& Mulligan, N. W. (1994). Theoretical implications of the mnemonic benefits of perceptual interference. Journal of Experimental Psychology: Learning, Memory, \& Cognition, 20, 608-620.

Holender, D. (1986). Semantic activation without conscious identification in dichotic listening, parafoveal vision, and visual masking: A survey and appraisal. Behavioral \& Brain Sciences, 9, 1-66.

JACOBY, L. L., \& DALlas, M. (1981). On the relationship between autobiographical memory and perceptual learning. Journal of Experimental Psychology: General, 110, 306-340.

JACoby, L. L., Levy, B. A., \& Steinbach, K. (1992). Episodic transfer and automaticity: Integration of data-driven and conceptually-driven processing in reading. Journal of Experimental Psychology: Learning, Memory, \& Cognition, 18, 15-24.

JACOBY, L. L., LindSAY, D. S., \& ToTH, J. P. (1992). Unconscious influences revealed: Attention, awareness, and control. American Psychologist, 47, 802-809.

JACoby, L. L., Toth, J. P., \& Yonelinas, A. P. (1993). Separating conscious and unconscious influences of memory: Measuring recollection. Journal of Experimental Psychology: General, 122, 139-154.

Kahneman, D., \& Treisman, A. (1984). Changing views of attention and automaticity. In R. Parasuraman \& D. R. Davies (Eds.), Varieties of attention (pp. 29-61). Orlando, FL: Academic Press.

MacDonald, P., \& MAcLeod, C. M. (1998). The influence of attention at encoding in direct and indirect remembering. Acta Psychologica, 98, 291-310.

Mack, A., \& Rock, I. (1998). Inattentional blindness. Cambridge, MA: MIT Press.

Masson, M. E. J., \& MacLeod, C. M. (1992). Reenacting the route to interpretation: Enhanced perceptual identification without prior perception. Journal of Experimental Psychology: General, 121, 145176.

McCann, R. Folk, C. L., \& Johnston, J. C. (1992). The role of spatial attention in visual word processing. Journal of Experimental Psychology: Human Perception \& Performance, 18, 1015-1029.

Mulligan, N. W. (1996). The effects of perceptual interference at encoding on implicit memory, explicit memory, and memory for source. Journal of Experimental Psychology: Learning, Memory, \& Cognition, 22, 1067-1087.

Mulligan, N. W. (1997). Attention and implicit memory tests: The effects of varying attentional load on conceptual priming. Memory \& Cognition, 25, 11-17.

Mulligan, N. W., \& Hartman, M. (1996). Divided attention and indirect memory tests. Memory \& Cognition, 24, 453-465.

Mulligan, N. W., \& Hornstein, S. L. (2000). Attention and perceptual priming in the perceptual identification task. Journal of Experimental Psychology: Learning, Memory, \& Cognition, 26, 626-637.

Neill, W. T., Beck, J. L., Bottalico, K. S., \& Molloy, R. D. (1990). Effects of intentional versus incidental learning on explicit and implicit tests of memory. Journal of Experimental Psychology: Learning, Memory, \& Cognition, 16, 457-463.

Parkin, A. J., ReID, T. K., \& Russo, R. (1990). On the differential nature of implicit and explicit memory. Memory \& Cognition, 18, $507-$ 514.

PARKIN, A. J., \& Russo, R. (1990). Implicit and explicit memory and the automatic/effortful distinction. European Journal of Cognitive Psychology, 2, 71-80.

RoEdiger, H. L., III (1990). Implicit memory: Retention without remembrance. American Psychologist, 45, 1043-1056.

Roediger,H. L., III, Weldon, M. S., Stadler, M. L., \& Riegler, G. L. (1992). Direct comparison of two implicit memory tests: Word fragment and word stem completion. Journal of Experimental Psychology: Learning, Memory, \& Cognition, 18, 1251-1269.

SCHNEIDER, W. (1988). Micro Experimental Laboratory: An integrated system for IBM PC compatibles. Behavior Research Methods, Instruments, \& Computers, 20, 206-217.

Schneider, W., Dumais, S. T., \& Shiffrin, R. M. (1984). Automatic and control processing and attention. In R. Parasuraman \& D. R. Davies (Eds.), Varieties of attention (pp. 1-27). Orlando, FL: Academic Press.

ShIFFrin, R. M. (1997). Attention, automatism, and consciousness. In J. D. Cohen \& J. W. Schooler (Eds.), Scientific approaches to consciousness (pp. 49-64). Mahwah, NJ: Erlbaum.

Shiffrin, R. M., \& SchneIder, W. (1977). Controlled and automatic human information processing: II. Perceptual learning, automatic encoding, and a general theory. Psychological Review, 84, 127-190.

Smith, M. E., \& Oscar-Berman, M. (1990). Repetition priming of words and pseudowords in divided attention and in amnesia. Journal of Experimental Psychology: Learning, Memory, \& Cognition, 16, 1033-1042.

Stone, M., Ladd, S. L., Vaidya, C. J., \& Gabrieli, J. D. E. (1998). Word-identification priming for ignored and attended words. Consciousness \& Cognition, 7, 238-258.

von Hippel, W., \& Hawkins, C. (1994). Stimulus exposure time and perceptual memory. Perception \& Psychophysics, 56, 525-535.

WELDON, M. S. (1991). Mechanisms underlying priming on perceptual tests. Journal of Experimental Psychology: Learning, Memory, \& Cognition, 17, 526-541.

Wood, N. L., Stadler, M. A., \& Cowan, N. (1997). Is there implicit memory without attention? A reexamination of task demands in Eich's (1984) procedure. Memory \& Cognition, 25, 772-779.

YANTIS, S., \& JoNidES, J. (1990). Abrupt visual onsets and selective attention: Voluntary versus automatic allocation. Journal of Experimental Psychology: Human Perception \& Performance, 16, 121-134. 


\section{NOTES}

1. Because of a programming error, for one half of the participants in each encoding condition, the displays on which target digits appeared was random, so that a target digit could occur adjacent to a critical word. Analyses showed no reliable effect of the programming error on encoding, recognition, or perceptual identification performance (all $p \mathrm{~s}>$ .24). Thus, the data were collapsed over the procedural error in the reported analyses.

2. A related possibility is that performance in the perceptual identification task was contaminated by an explicit strategy: The participants may have noticed that some of the words in the perceptual identification task were old, and they may have tried to respond with old words. However, we found no evidence for contamination in our data. Following a strategy used by others, we compared the level of perceptual implicit memory on the first half of the perceptual identification task with the level of perceptual implicit memory on the second half of the task. Explicit strategies take time to develop, so support for contamination would be finding higher levels of perceptual implicit memory in the second half of the trials. An ANOVA on the difference scores, with trial half as a within-subjects variable and encoding condition as a betweensubjects variable showed no significant effects, although the mean level of perceptual implicit memory was numerically lower for the second half of the trials for both the digit and the word conditions (.127 for the first half and .100 for the second half). A similar analysis on the data from Experiment 2 also showed no significant effects.

3. Hirshman and Mulligan (1991) also found an effect of duration on explicit memory and no effect on perceptual implicit memory, but they found that shorter durations led to higher levels of explicit memory, the opposite of the present findings. Their shorter durations were much shorter than ours ( $100 \mathrm{msec}$ or less), and the words were masked. Hirshman, Trembath, and Mulligan (1994) and Mulligan (1996) suggested that perceptual interference at encoding (short duration and a mask) facilitated some type of item-specific processing not required for perceptual implicit memory. The results of Experiment 2 suggest that with durations of at least $200 \mathrm{msec}$ and no mask, there is no facilitation.

4. A reviewer noted that the lack of an exposure duration effect might be due to the insensitivity of the perceptual identification task. That is, the results might be due to only one kind of controlled processing (affected by both manipulations), but the presentation duration manipulation was too weak to affect performance on the perceptual identification task.

(Manuscript received September 13, 1999; revision accepted for publication February 19, 2001.) 